\title{
MicroRNAs in lipid metabolism
}

\author{
Carlos Fernández-Hernando, Yajaira Suárez, Katey J. Rayner, and Kathryn J. Moore \\ Departments of Medicine and Cell Biology, Leon H. Charney Division of Cardiology and the Marc \\ and Ruti Bell Vascular Biology and Disease Program, New York University School of Medicine, \\ New York, New York, USA
}

\begin{abstract}
Purpose of review-Although the role for microRNAs (miRNAs) in regulating multiple physiological processes including apoptosis, cell differentiation, and cancer is well established, the importance of these tiny RNAs in regulating lipid metabolism has only recently been uncovered. This review summarizes the evidence for a critical role of miRNAs in regulating lipid metabolism.

Recent findings-Lipid metabolism is tightly regulated at the cellular level. In addition to classic transcriptional regulation of cholesterol metabolism (e.g. by SREBP and LXR), members of a class of noncoding RNAs termed miRNAs have now been identified to be potent posttranscriptional regulators of lipid metabolism genes involved in cholesterol homeostasis and fatty acid oxidation. Several reports have recently shown that miR-33 regulates cholesterol efflux and HDL biogenesis by downregulating the expression of the ABC transporters, ABCA1 and ABCG1. In addition, miR-33 also inhibits the translation of several transcripts encoding proteins involved in fatty acid $\beta$-oxidation including CPT1a, CROT, and HADHB, thereby reducing fatty acid degradation. Other miRNAs including miR-122, miR-370, miR-335, and miR-378/378*, miR-27 and miR-125a-5p have been implicated in regulating cholesterol homeostasis, fatty acid metabolism and lipogenesis.
\end{abstract}

Summary-Recent advances in the understanding of the regulation of lipid metabolism indicate that miRNAs play major roles in regulating cholesterol and fatty acid metabolism. These new findings may open new avenues for the treatment of dyslipidemias.

\section{Keywords}

cholesterol homeostasis; HDL; microRNAs

\section{Introduction}

Despite advances in the prevention and management of cardiovascular disease (CVD), this multifactorial disorder remains a leading cause of mortality worldwide. Insufficient or excessive cellular cholesterol results in pathologic processes including atherosclerosis and metabolic syndrome [1-4]. The mechanisms controlling this balance remain incompletely understood. MicroRNAs (miRNA) are short ( $22 \mathrm{nt})$, double-stranded RNAs encoded in the genome that bind to complementary target sites in the $3^{\prime}$ untranslated regions ( $3^{\prime}$ UTR) of mRNAs, causing translational repression and/or mRNA destabilization [5-7]. Notably, a single miRNA can have multiple targets, thus providing a mechanism for simultaneously regulating the post-transcriptional expression of genes involved in a specific pathway or

(C) 2011 Wolters Kluwer Health | Lippincott Williams \& Wilkins

Correspondence to Carlos Fernández-Hernando, Departments of Medicine and Cell Biology, Leon H. Charney Division of Cardiology and the Marc and Ruti Bell Vascular Biology and Disease Program, New York University School of Medicine, New York, NY 10016, USA Tel: +1 212263 9324; carlos.fernandez-hernando@nyumc.org. 
physiological process [5-7]. The specific role of miRNAs in regulating lipid metabolism is a burgeoning area of investigation. In this review, we will summarize the current knowledge of miRNAs involved in the regulation of lipid metabolism. We will specifically focus on the contribution of miR-33a and miR-33b, intronic miRNAs, in the epigenetic regulation of cholesterol homeostasis and fatty acid metabolism.

\section{Regulation of cholesterol and fatty acid metabolism}

Lipid homeostasis in mammals is regulated by a family of membrane-bound transcription factors called sterol regulatory element-binding proteins (SREBPs) [8-10]. SREBPs directly activate the expression of more than 30 genes involved in the synthesis and uptake of cholesterol, fatty acids, triglycerides, and phospholipids, as well as the NADPH cofactor required to synthesize these molecules [8-10]. The mammalian genome encodes three SREBP isoforms, designated SREBP-1a, SREBP-1c, and SREBP-2. The SREBPs differ in their tissue-specific expression, their target gene selectivity and the relative potencies of their trans-activation domains. Specific analyses of individual isoforms suggest that SREBP-1 is selectively involved in activating genes involved in fatty acid metabolism and de-novo lipogenesis, whereas SREBP-2 is more selective for genes involved directly in cholesterol homeostasis [8-10] (Fig. 1). Aberrant expression of SREBPs in mice results in metabolic syndromes with physiological effects similar to specific disorders of lipid metabolism in humans [8-10].

In addition to SREBP, the liver X receptors (LXRs) are also important transcriptional regulators of cholesterol metabolism [11,12]. LXR $\alpha$ (NR1H3) and LXR $\beta$ (NR1H2) nuclear receptors form heterodimers with retinoid $X$ receptors and are activated by a variety of sterols including oxysterol intermediates that form during cholesterol biosynthesis [12]. LXRs activate the transcription of genes involved in cellular cholesterol efflux including $A b c a 1$ and Abcgl [12]. Mice lacking LXRs accumulate sterols in their tissues and manifest accelerated atherosclerosis [13], whereas synthetic LXR agonists promote reverse cholesterol transport and protect mice against atherosclerosis [14,15]. LXRs also impact SREBP-1 transcription, thereby mediating cross-talk between these pathways to increase fatty acid synthesis [16]. Mice with a targeted disruption in the LXRA gene were noted to be deficient in expression of SREBP-1c, fatty acid synthase (FAS), SCD-1 (steroyl CoA desaturase I), and ACC (acyl CoA carboxylase) [16,17]. By contrast, pharmacological activation of LXR triggers induction of the lipogenic pathway and elevates plasma triglyceride levels leading to hepatic steatosis in mice [18]. Together these pathways contribute majorly to the transcriptional regulation of lipid homeostasis.

\section{MicroRNAS}

MicroRNAs (miRNAs) are small ( $22 \mathrm{nt}$ ), single-stranded, noncoding RNAs, which were first discovered in the nematode Caenorhabditis elegans [19,20]. miRNAs are encoded in the genomes of most eukaryotes and some viruses [5-7,21]. Mature miRNAs are derived from precursors called pri-miRNA composed of hundreds or thousands of nucleotides that constitute monocistronic or polycistronic transcriptional units [5-7,21]. Notably, miRNAs can be located within introns or exons of protein-coding genes. The primary transcript or pri-miRNA is usually transcribed by RNA polymerase II and then processed by the action of Drosha along with its cofactor DGCR8 to produce a partially processed hairpin precursor (pre-miRNA) in the nucleus. The pre-miRNA is then exported to the cytoplasm by Exportin 5 , where it undergoes to the next processing step mediated by another RNase, Dicer, to produce the mature miRNA. After the cleavage, one strand of the miRNA duplex is preferentially incorporated into the RISC complex where it associates with Argonaute proteins directing the binding of the RISC complex to partially complementary sites in the $3^{\prime}$ 
UTR of mRNAs. Association of the RISC complex to the specific mRNA mediates its repression, either by transcript destabilization, translational inhibition, or both. WatsonCrick base pairing between the target and the 5'-end of miRNAs, the 'seed' sequence (nt 27 ), is an important determinant of functional target sites. However, absence of perfect complementarity between the miRNA sequence and target sites can complicate the identification of miRNA target genes. Reviews covering these topics and more general information about biogenesis and mechanisms of miRNA action are widely available [5$7,21]$.

\section{MicroRNAs and lipid metabolism}

To date several miRNAs have been described to regulate lipid metabolism including miR-122, miR-370, miR-378/378*, miR-335, miR-125a-5p, and miR-33. miR-122 was initially identified as a highly abundant miRNA in the liver, accounting for $70 \%$ of total miRNA expression [22]. Orthologs in numerous vertebrate species have been reported and the liver specificity appears to be conserved. miR-122 has been associated with the regulation of liver metabolism as well as hepatitis $\mathrm{C}$ infection, and has been shown to be downregulated in hepatocellular carcinoma (HCC) $\left[23,24,25^{\circ}\right]$. The use of antisense miR-122 downregulated several genes implicated in liver metabolism and produced an increase in expression of hundreds of genes that are normally repressed in hepatocytes, suggesting that miR-122 functions to maintain the liver phenotype [23,24]. In addition, several genes involved in fatty acid synthesis and oxidation were altered in mice treated with anti-miR-122 including FAS, ACC1, and ACC2 [23,24]. Furthermore, silencing of miR-122 in high-fat fed mice reduced hepatic steatosis, which was linked to a reduction in cholesterol synthesis and stimulation of fatty-acid oxidation [24].

A second miRNA, miR-370 was recently shown to have similar effects on lipid metabolism as miR-122. miR-370 targets carnitine palmitoyl transferase (Cpt1a), a mitochondrial enzyme that mediates the transport of long-chain fatty acids across the membrane by binding them to carnitine, thereby reducing fatty acid oxidation [26]. Notably, transfection of the human hepatic cell line HepG2 with miR-370, upregulates the expression of miR-122 leading to an increased expression of lipogenic genes including SREBP1c and DGAT2, suggesting that miR-370 provides an additional point of regulation of this pathway [26].

miR-378/378*, an intronic miRNA located within the peroxisome proliferator-activated receptor gamma coactivator-1 alpha (PGC1 $\alpha$ ) genomic sequence, also plays important roles in regulating lipid metabolism [ $\left.27^{\circ}\right]$. Overexpression of miR-378/378* during adipogenesis increases triacylglycerol accumulation $\left[27^{\circ}\right]$. Adipocyte ST2 cells transfected with miR-378/378* show increased expression of fatty acid metabolism genes including fatty acid binding protein 4 (FABP4), FAS, and stearoyl-coenzymeA desaturase (SCD-1) [27 ${ }^{\circ}$. In addition to miR-378/378*, miR-143, miR-27, and miR-335 have also been recently associated with lipid metabolism and adipocyte differentiation $\left[28,29,30^{\circ}\right]$. The introduction of antisense oligonucleotides against miR-143 into preadipocytes inhibited their differentiation by downregulating ERK5, indicating a role for this microRNA in adipocyte differentiation [28]. By contrast, miR-27 inhibits the expression of PPAR $\gamma$ and C/EBPa and is downregulated during adipocyte differentiation [30 $\left[0^{\circ}\right.$. miR-335 is upregulated in response to lipid loading and is highly expressed in liver and adipose tissue of obese mice [31]. However, the role of miR-335 in regulating lipid metabolism and adipogenesis remains unknown.

\section{miR-33, a key regulator of lipid metabolism}

miR-33a is an intronic miRNA located within intron 16 of human SREBF-2, a master switch in controlling genes involved in cholesterol uptake and synthesis. Despite the report of its 
genomic location in 2004, it would be a number of years before the significance and the functional consequences of this location would become apparent. However, in a series of parallel yet independent studies, miR-33 was identified as a key post-transcriptional regulator of cellular cholesterol homeostasis $\left[32^{* \bullet}-34^{\circ \bullet}\right]$. In a study from our group, profiling of over 300 microRNAs that were altered by cholesterol content in human macrophages revealed that miR-33a was upregulated in response to sterol depletion [32*0]. Due to its intriguing intronic location within Srebf-2 gene, we chose this miRNA for further study (Fig. 2). We demonstrated that in both normal and hypercholesteromic mice, dietary cholesterol alters miR-33 expression levels in both the liver and peritoneal macrophages. NajafiShoushtari et al. and Marquart et al. came upon miR-33 by very different means: they were interested in further understanding how transcription factors such as the SREBPs control cholesterol gene regulation, and in silico bioinformatic assessment of these loci uncovered the presence of a highly conserved miRNA (miR-33) within an intron of Srebf-2 $\left[33^{* *}, 34^{* *}\right]$. Najafi-Shoustari et al. [34**] also pointed out the presence of a second isoform of miR-33: $m i R-33 b$ which is found within Srebf-1. Studies from these groups showed that $m i R-33 a$ is co-transcribed along with Srebf-2 in both hepatocytes and macrophages, during states of cholesterol depletion, and that the expression of miR-33a and Srebf-2 is comparable across many tissues. Moreover, Horie et al. [35"*] recently cloned the exon 16 through exon 17 of human SREBP-2, including the intron containing $m i R-33 a$, and showed that $m i R-33 a$ is indeed expressed when expression of Srebf-2 is activated. Furthermore, the presence of $m i R$-33a within Srebf-2 is highly conserved from mammals to Drosophila, implying that this miRNA may be performing a specific and important function that has been conserved under evolutionary pressure.

\section{miR-33 targets genes involved in cholesterol transport}

Due to its regulation both in vitro and in vivo by cellular cholesterol status, it perhaps was no surprise that among the top-predicted gene targets of miR-33 was ABCA1 - a transporter responsible for the movement of cholesterol out of the cell. The 3' UTR of Abcal contains three highly conserved binding sites for miR-33a and/or miR-33b. Indeed, miR-33a strongly represses the expression of ABCA1 mRNA and protein levels in a variety of cell types $\left[32^{* *}-34^{*}\right]$. Analysis of the $3^{\prime}$ UTR of Abcal reveals that the targeting by miR-33 is direct and specific, and mutations in these sites relieves of the repression of $A b c l$ by miR-33 [32*$34^{* *}$. Functionally, overexpression of miR-33 in macrophages results in a decrease of cholesterol efflux to apoA1 - the first step in the generation of nascent HDL particles. Perhaps more importantly however, the inhibition of endogenous miR-33 results in an increase in expression of ABCA1 protein, and a concomitant increase in cholesterol efflux to apoA1, indicating that this miRNA is indeed mediating the repression of its target genes at physiological levels $\left[32^{* *}-34^{* *}\right]$. Taken together, these results demonstrate that ABCA1 is under the post-transcriptional control of miR-33, and that the inhibition of this pathway in vivo, leads to increased ABCA1 expression and cholesterol efflux.

In addition to ABCA1, we identified two other genes involved in cholesterol mobilization in the cell as targets of miR-33: ABCG1 $\left[32^{* *}, 33^{* *}\right]$, which mobilizes cellular-free cholesterol to more mature HDL particles for efflux, and NPC1 [32*日), which transports cholesterol from lysosomal compartments to other parts of the cell in need (Fig. 2). In the mouse genome, the 3' UTR of Abcgl contains two miR-33 binding sites; however, these sites are not conserved in the human $3^{\prime}$ UTR. This results in the inhibition of $A B C G 1$ expression by miR-33 in mouse cells, but not in cells of human origin $\left[32^{* *}, 33^{\circ *}\right]$. The functional consequence of ABCG1 targeting by miR-33 was demonstrated by a decrease in cholesterol efflux to HDL after overexpression of miR-33 in cells of mouse but not human origin [32**]. The $3^{\prime}$ UTR of $\mathrm{Npcl}$ in humans contains two miR-33 binding sites, resulting in repression of NPC1 protein expression by miR-33, whereas the mouse only contains one site [32*0]. NPC1 acts together 
with ABCA1 to efflux cholesterol to apoA1 [36], indicating that miR-33 represses a second part of the cellular cholesterol export pathway. These results indicate that miR-33 controls cholesterol export through the coordinated regulation of ABCA1, NPC1, and ABCG1 which regulate the efflux of cholesterol to the reverse cholesterol transport pathway.

\section{miR-33 regulation of $\mathrm{HDL}$ in vivo}

The observations that miR-33 could reciprocally control ABCA1 and ABCG1 expression upon activation of SREBP-2 led to the hypothesis that miR-33 may be controlling HDL levels in vivo. Using a variety of approaches, from viral delivery of antisense oligonucleotides to locked nucleic acid (LNA) inhibitors, it was demonstrated that inhibition of miR-33 results in an increase in hepatic ABCA1 protein expression and a resultant increase in circulating HDL $\left[32^{* *}-34^{*}\right]$. These experiments were performed in both normal and high-fat diet-fed mice, indicating that even when miR-33 levels would be predictably low, inhibition of miR-33 can still result in a functional increase in HDL. Following these

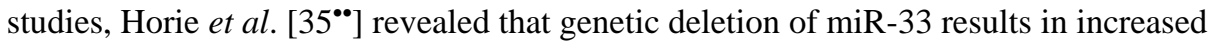
hepatic ABCA1 expression and a $25 \%$ increase in circulating HDL levels, confirming the

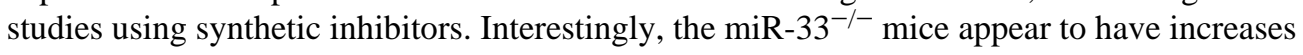
in the larger HDL fractions, with no changes observed in the smaller HDL fractions [35"*]. This is similar to what was observed by other groups using miR-33 inhibitors, showing increases in HDL cholesterol in the larger fractions by FPLC [32*-34**]. This increase in HDL particle size may be as a result of efflux of cholesterol to HDL from extrahepatic tissues into the reverse cholesterol transport pathway, confirming that miR-33 targeting of ABCA1 and ABCG1 play important roles not only in the liver but in other tissues as well.

\section{miR-33 targets genes involved in fatty acid metabolism}

In addition to the cholesterol transport genes ABCA1, ABCG1, and NPC1, we and Davalos et al. (unpublished data) showed that miR-33 binding sites are highly conserved in the $3^{\prime}$

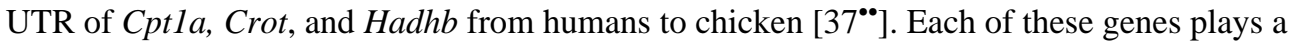
distinct role in the fatty acid oxidation pathway: CPT1a is required for coupling of acetyl CoA to carnitine, allowing for transport of medium-chain and long-chain fatty acids to the mitochondria for $\beta$-oxidation [38-40]. CPT1a is the rate-limiting step for this process, and is not surprisingly one of the most highly regulated members of the $\beta$-oxidation pathway [3840]. Similarly, CROT is a peroxisomal enzyme that is required for the coupling of shortchain fatty acids to carnitine, also transporting them into the mitochondria. Finally, HADHB is required for the last three steps in the $\beta$-oxidation pathway in the mitochondria [38-40]. Through analysis of the 3' UTR activity of Cptla, Crot, and Hadhb, we showed that miR-33 indeed targets each of these members of the fatty acid oxidation pathway, and overexpression of miR-33 led to decreased CPT1a and HADHB expression, and reduced cellular fatty acid $\beta$-oxidation. Intriguingly, Gerin et al. [37"*] focused their studies exclusively on miR-33a, and did not examine the effects of miR-33b. Gerin et al. speculate that when SREBP-2 is activated, miR-33a could also target genes involved in cellular $\beta$ oxidation of fatty acids, which is known to occur during states of cholesterol. However, miR-33b is encoded within Srebp-1, which would be predictably upregulated during states of insulin excess, when cellular fatty acid $\beta$-oxidation would be reduced. Indeed, we saw that activation of SREBP-1 with an LXR agonist leads to increased expression of miR-33b (Davalos et al., unpublished data). However, given that miR-33a and miR-33b differ by only two nucleotides, it is likely that miR-33b is capable of reducing expression of the fatty acid oxidation machinery and reduces $\beta$-oxidation similar to miR-33a. Indeed, we have recently found that miR-33a and miR-33b similarly target the 3' UTR activity of Abcal, Crot, Cptla, $H a d h b$, and $A m p k \alpha$, indicating that the two-nucleotide variation in the mature forms of miR-33a and miR-33b does not appreciably affect the gene targeting by these miRNAs 
(Davalos et al., unpublished data). Unfortunately, the in-vivo analysis of miR-33b will be challenging owing to the lack of conservation of this miRNA in lower mammals such as mice.

\section{Conclusion}

miRNAs represent an elegant layer above transcriptional control for both fine-tuning and dramatically altering activity and output of cell signaling. In addition, miRNAs may serve as points of cross talk between signaling pathways, by integrating transcriptional inputs or by their functional regulatory output on different pathways. The results summarized herein show the promise for the use of anti-miR33 therapies to treat a number of metabolic disorders. Using anti-miR33 treatment to elevate ABCA1 levels and increase HDL levels would hold tremendous potential for the treatment and/or prevention of coronary artery disease (CAD), in which an underlying risk factor is low levels of HDL. Moreover, inhibition of miR-33 would result in increased fatty acid oxidation and reduced accumulation of fat stores in the liver. Given the promise demonstrated by the use of antimiRs in preclinical studies, it is possible that miR-33 may become a viable therapeutic target in the future.

\section{Acknowledgments}

C.F.-H. laboratory is supported by grants from American Heart Association (SDG-0835585D) and National Institute of Health (1P30HL101270-01), the Y.S. laboratory is supported by grant from American Heart Association (SDG-0835481N) and the K.J.M. laboratory is supported by the National Institute of Health (R01AG02055). We apologize to those whose work could not be cited owing to space limitations.

\section{References and recommended reading}

Papers of particular interest, published within the annual period of review, have been highlighted as:

- of special interest

•• of outstanding interest

Additional references related to this topic can also be found in the Current World Literature section in this issue (pp. 137-138).

1. Alberti KG, Zimmet P, Shaw J. Metabolic syndrome: a new world-wide definition. A Consensus Statement from the International Diabetes Federation. Diabet Med. 2006; 23:469-480. [PubMed: 16681555]

2. Glass CK, Witztum JL. Atherosclerosis. The road ahead. Cell. 2001; 104:503-516. [PubMed: 11239408]

3. Lusis AJ. Atherosclerosis. Nature. 2000; 407:233-241. [PubMed: 11001066]

4. Teran-Garcia M, Bouchard C. Genetics of the metabolic syndrome. Appl Physiol Nutr Metab. 2007; 32:89-114. [PubMed: 17332787]

5. Ambros V. MicroRNA pathways in flies and worms: growth, death, fat, stress, and timing. Cell. 2003; 113:673-676. [PubMed: 12809598]

6. Ambros V. The functions of animal microRNAs. Nature. 2004; 431:350-355. [PubMed: 15372042]

7. Bartel DP. MicroRNAs: target recognition and regulatory functions. Cell. 2009; 136:215-233. [PubMed: 19167326]

8. Brown MS, Goldstein JL. The SREBP pathway: regulation of cholesterol metabolism by proteolysis of a membrane-bound transcription factor. Cell. 1997; 89:331-340. [PubMed: 9150132]

9. Horton JD, Goldstein JL, Brown MS. SREBPs: activators of the complete program of cholesterol and fatty acid synthesis in the liver. J Clin Invest. 2002; 109:1125-1131. [PubMed: 11994399] 
10. Osborne TF. Sterol regulatory element-binding proteins (SREBPs): key regulators of nutritional homeostasis and insulin action. J Biol Chem. 2000; 275:32379-32382. [PubMed: 10934219]

11. Peet DJ, Janowski BA, Mangelsdorf DJ. The LXRs: a new class of oxysterol receptors. Curr Opin Genet Dev. 1998; 8:571-575. [PubMed: 9794827]

12. Tontonoz P, Mangelsdorf DJ. Liver X receptor signaling pathways in cardiovascular disease. Mol Endocrinol. 2003; 17:985-993. [PubMed: 12690094]

13. Bradley MN, Hong C, Chen M, et al. Ligand activation of LXR beta reverses atherosclerosis and cellular cholesterol overload in mice lacking LXR alpha and apoE. J Clin Invest. 2007; 117:23372346. [PubMed: 17657314]

14. Claudel T, Leibowitz MD, Fievet C, et al. Reduction of atherosclerosis in apolipoprotein E knockout mice by activation of the retinoid X receptor. Proc Natl Acad Sci U S A. 2001; 98:26102615. [PubMed: 11226287]

15. Joseph SB, McKilligin E, Pei L, et al. Synthetic LXR ligand inhibits the development of atherosclerosis in mice. Proc Natl Acad Sci U S A. 2002; 99:7604-7609. [PubMed: 12032330]

16. Repa JJ, Liang G, Ou J, et al. Regulation of mouse sterol regulatory element-binding protein-1c gene (SREBP-1c) by oxysterol receptors, LXRalpha and LXRbeta. Genes Dev. 2000; 14:28192830. [PubMed: 11090130]

17. Peet DJ, Turley SD, Ma W, et al. Cholesterol and bile acid metabolism are impaired in mice lacking the nuclear oxysterol receptor LXR alpha. Cell. 1998; 93:693-704. [PubMed: 9630215]

18. Chen G, Liang G, Ou J, et al. Central role for liver X receptor in insulin-mediated activation of Srebp-1c transcription and stimulation of fatty acid synthesis in liver. Proc Natl Acad Sci U S A. 2004; 101:11245-11250. [PubMed: 15266058]

19. Lee RC, Feinbaum RL, Ambros V. The C. elegans heterochronic gene lin-4 encodes small RNAs with antisense complementarity to lin-14. Cell. 1993; 75:843-854. [PubMed: 8252621]

20. Wightman B, Ha I, Ruvkun G. Posttranscriptional regulation of the heterochronic gene lin-14 by lin-4 mediates temporal pattern formation in C. elegans. Cell. 1993; 75:855-862. [PubMed: 8252622]

21. Filipowicz W, Bhattacharyya SN, Sonenberg N. Mechanisms of posttranscriptional regulation by microRNAs: are the answers in sight? Nat Rev Genet. 2008; 9:102-114. [PubMed: 18197166]

22. Lagos-Quintana M, Rauhut R, Yalcin A, et al. Identification of tissue-specific microRNAs from mouse. Curr Biol. 2002; 12:735-739. [PubMed: 12007417]

23. Elmen J, Lindow M, Schutz S, et al. LNA-mediated microRNA silencing in nonhuman primates. Nature. 2008; 452:896-899. [PubMed: 18368051]

24. Esau C, Davis S, Murray SF, et al. miR-122 regulation of lipid metabolism revealed by in vivo antisense targeting. Cell Metab. 2006; 3:87-98. [PubMed: 16459310]

$25 \bullet$. Lanford RE, Hildebrandt-Eriksen ES, Petri A, et al. Therapeutic silencing of microRNA-122 in primates with chronic hepatitis C virus infection. Science. 2010; 327:198-201. [PubMed: 19965718] This study establishes that treatment of chronically infected chimpanzees with a LNA-modified oligonucleotide complementary to miR-122 leads to long-lasting suppression of $\mathrm{HCV}$ viremia and shows the promise of anti-miR targeted therapy for the treatment of chronic diseases.

26. Iliopoulos D, Drosatos K, Hiyama Y, et al. MicroRNA-370 controls the expression of microRNA-122 and Cpt1alpha and affects lipid metabolism. J Lipid Res. 2010; 51:1513-1523. [PubMed: 20124555]

$27 \bullet$ Gerin I, Bommer GT, McCoin CS, et al. Roles for miRNA-378/378* in adipocyte gene expression and lipogenesis. Am J Physiol Endocrinol Metab. 2010; 299:E198-E206. [PubMed: 20484008] This analysis of microRNAs in adipocyte metabolism revealed that miR-378/378*, encoded within PGC-1 $\beta$, controls the expression of a set of liponic genes.

28. Esau C, Kang X, Peralta E, et al. MicroRNA-143 regulates adipocyte differentiation. J Biol Chem. 2004; 279:52361-52365. [PubMed: 15504739]

29. Kajimoto K, Naraba H, Iwai N. MicroRNA and 3T3-L1 preadipocyte differentiation. RNA. 2006; 12:1626-1632. [PubMed: 16870994]

$30 \bullet$. Lin Q, Gao Z, Alarcon RM, et al. A role of miR-27 in the regulation of adipogenesis. FEBS J. 2009; 276:2348-2358. [PubMed: 19348006] This paper reports that miR-27, which is increased 
in the adipose of obese mice, controls adipogenesis by reducing expression of PPAR- $\gamma$ and c/ $\operatorname{EBP} \alpha$.

31. Nakanishi N, Nakagawa Y, Tokushige N, et al. The up-regulation of microRNA-335 is associated with lipid metabolism in liver and white adipose tissue of genetically obese mice. Biochem Biophys Res Commun. 2009; 385:492-496. [PubMed: 19460359]

$32 \bullet$. Rayner KJ, Suarez Y, Davalos A, et al. MiR-33 contributes to the regulation of cholesterol homeostasis. Science. 2010; 328:1570-1573. [PubMed: 20466885] This study reports that miR-33a, an intronic miRNA located within the SREBF-2 gene, is regulated by cellular and dietary cholesterol and coordinately inhibits a number of key genes involved in cellular cholesterol transport, including ABCA1, ABCG1 and NPC1. The authors show that lentiviral silencing of miR-33 in vivo increases plasma HDL levels, and together with Refs. [33 ${ }^{\bullet \bullet}, 34^{\bullet \bullet}$ ], establish miR-33 as a regulator of cholesterol homeostasis.

33 •. Marquart TJ, Allen RM, Ory DS, Baldan A. miR-33 links SREBP-2 induction to repression of sterol transporters. Proc Natl Acad Sci U S A. 2010; 107:12228-12232. [PubMed: 20566875] This study demonstrates that miR-33a, an intronic miRNA located within the SREBF-2 gene, inhibits two members of the ABC transporter family, ABCA1 and ABCG1, that regulate cellular cholesterol efflux. The authors show that adenoviral-mediated silencing of miR-33 in vivo increases plasma HDL levels, and together with Refs. $\left[32^{\bullet \bullet}, 34^{\bullet \bullet}\right]$, establish miR-33 as a regulator of cholesterol homeostasis.

34 ••. Najafi-Shoushtari SH, Kristo F, Li Y, et al. MicroRNA-33 and the SREBP host genes cooperate to control cholesterol homeostasis. Science. 2010; 328:1566-1569. [PubMed: 20466882] This work shows that miR-33a and miR-33b act in concert with their SREBP host genes to regulate expression of ABCA1 and cholesterol efflux to apoA1. The authors show that LNA-mediated

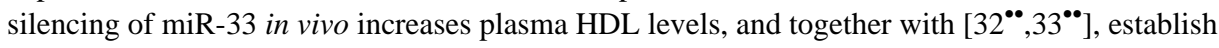
miR-33 as a regulator of cholesterol homeostasis.

$35 \bullet \bullet$. Horie T, Ono K, Horiguchi M, et al. MicroRNA-33 encoded by an intron of sterol regulatory element-binding protein 2 (Srebp2) regulates HDL in vivo. Proc Natl Acad Sci U S A. 2010; 107:17321-17326. [PubMed: 20855588] This study reports the generation of a miR-33 knockout mouse and establishes a critical role for miR-33 in the regulation of ABCA1 expression and HDL biogenesis in vivo.

36. Wang MD, Franklin V, Sundaram M, et al. Differential regulation of ATP binding cassette protein A1 expression and ApoA-I lipidation by Niemann-Pick type $\mathrm{C} 1$ in murine hepatocytes and macrophages. J Biol Chem. 2007; 282:22525-22533. [PubMed: 17553802]

$37 \bullet$. Gerin I, Clerbaux LA, Haumont O, et al. Expression of miR-33 from an SREBP2 intron inhibits cholesterol export and fatty acid oxidation. J Biol Chem. 2010; 285:33652-33661. [PubMed: 20732877] This report shows that in addition to cholesterol transport genes, miR-33a inhibits the expression of genes involved in fatty acid metabolism (CROT, CPT1a, HADHP). These data link the activation of SREBP2 with the repression of fatty acid $\beta$-oxidation.

38. Brady PS, Ramsay RR, Brady LJ. Regulation of the long-chain carnitine acyltransferases. FASEB J. 1993; 7:1039-1044. [PubMed: 8370473]

39. Mannaerts GP, Van Veldhoven PP, Casteels M. Peroxisomal lipid degradation via beta- and alphaoxidation in mammals. Cell Biochem Biophys. 2000; 32:73-87. [PubMed: 11330072]

40. Ramsay RR, Gandour RD. Selective modulation of carnitine long-chain acyltransferase activities. Kinetics, inhibitors, and active sites of COT and CPT-II. Adv Exp Med Biol. 1999; 466:103-109. [PubMed: 10709633] 


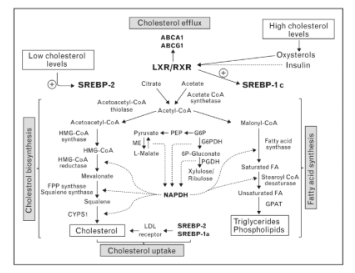

Figure 1. Gene regulatory network of lipid homeostasis In cholesterol-depleted conditions, SREBP-2 is activated and enhances the expression of genes dedicated to the synthesis and uptake of cholesterol, as well as the NADPH cofactor required to synthesize these molecules. Similarly, SREBP-1a regulates cholesterol metabolism but its expression is relatively low in vivo. SREBP-1c is activated by liver X receptor (LXR) agonists (oxysterols) and insulin, and regulates the expression of genes required for fatty acid synthesis. In cholesterol saturating conditions, the oxidized cholesterol derivatives activate LXR leading to an increase of ABCA1 and ABCG1 expression and cellular cholesterol efflux. 


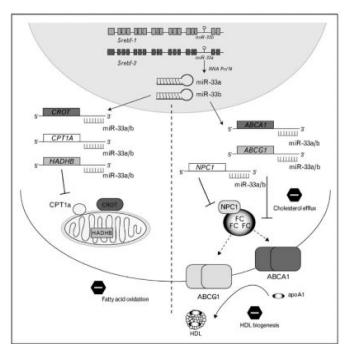

Figure 2. miR-33 represses expression of genes involved in cholesterol export and fatty acid oxidation

Activation of Srebf-1 or Srebf-2 results in co-transcription of miR-33b and miR-33a, respectively. These miRNAs simultaneously inhibit the expression of genes involved in fatty acid metabolism (CROT, CPT1a, HADHB) and cholesterol transport (ABCA1, ABCG1, NPC1). The outcome of miR-33a/b activation is reduced fatty acid $\beta$-oxidation and decreased movement of cholesterol into the HDL pathway. 